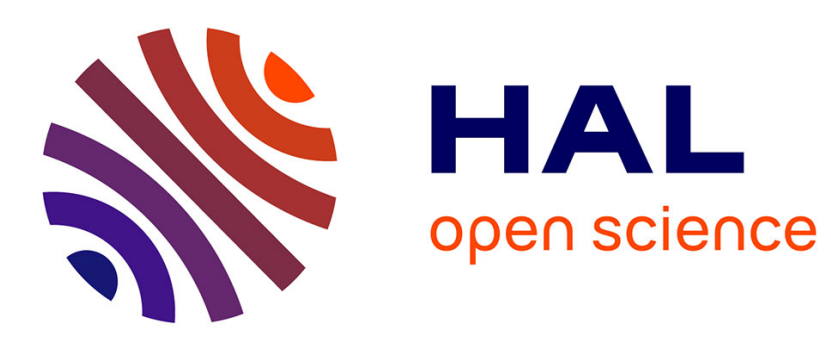

\title{
H\&HIL: A novel tool to test control strategy with Human and Hardware In the Loop
}

Bruno Jeanneret, Daniel Ndiaye, Sylvain Gillet, Rochdi Trigui

\section{To cite this version:}

Bruno Jeanneret, Daniel Ndiaye, Sylvain Gillet, Rochdi Trigui. H\&HIL: A novel tool to test control strategy with Human and Hardware In the Loop. VPPC 2017 (Vehicle Power and Propulsion Conference), Dec 2017, BELFORT, France. hal-01764553

\section{HAL Id: hal-01764553 \\ https://hal.science/hal-01764553}

Submitted on 12 Apr 2018

HAL is a multi-disciplinary open access archive for the deposit and dissemination of scientific research documents, whether they are published or not. The documents may come from teaching and research institutions in France or abroad, or from public or private research centers.
L'archive ouverte pluridisciplinaire HAL, est destinée au dépôt et à la diffusion de documents scientifiques de niveau recherche, publiés ou non, émanant des établissements d'enseignement et de recherche français ou étrangers, des laboratoires publics ou privés. 


\title{
H\&HIL: A novel tool to test control strategy with Human and Hardware In the Loop
}

\author{
Bruno Jeanneret, Daniel Ndiaye, Sylvain Gillet and Rochdi Trigui \\ IFSTTAR \\ 25 Av. François Mitterrand \\ 69675 Bron - France \\ Email: bruno.jeanneret@ifsttar.fr
}

\begin{abstract}
With this work, the authors try to make HIL simulation more realistic with the introduction of the Human driver in the loop. To succeed in this objective we develop a set of tools to connect easily a wide variety of real or virtual devices together : of course the driver but also racing gamer joystick, real engine, virtual drivetrain, virtual driver environment ...
\end{abstract}

The detailed approach represent a step forward before testing control strategy or new powertrain on a real vehicle. First results showed a good effectiveness and modularity of the tool.

\section{INTRODUCTION}

The transportation sector is responsible for a wide share of Energy consumption and pollutant emission everywhere in the world. The growth of environmental awareness is today among the most constringent drivers that the researchers and the manufacturers have to consider when designing environmental friendly solutions for drive trains, including electric, hybrid and fuel cell Vehicles. Nevertheless, most of the development and the evaluation of the new solutions still follow classical scheme that includes modeling and testing components and drivetrain under standard driving cycles or in the best case, using preregistered real world driving cycle [1], [2], [3], [4], [5] and [6]. This methodology of design does not include the variability of the driving conditions generated by the two major factors that are the driver behavior and the infrastructure influence (elevation, turns, speed limitation, traffic jam, traffic lights, ...).

Moreover, the progress registered in the Intelligent Transportation Systems (ITS) makes it possible today to optimize the actual use of the vehicles (classical, EVs, HEVs) by capturing instantaneous information about the infrastructure (road profile, road signs), the traffic and also about the weather conditions. These informations are used for HEV and PHEVs to optimize their energy management and for mono-sources vehicles (classical, EV) to give inputs to the ADAS systems in order to perform a lower consumption and emission (ecodriving concepts for example). The simulation and testing using standard or preregistered driving cycles is therefore too limited to take into account all these aspects. The need is then to develop new simulation and testing schemes able to consider a more realistic modeling of the vehicle environment and to include the driver or its model in the simulation/testing loop.

The methodology presented in this paper is based on a progressive and modular approach for simulating and testing in a HIL configuration different types of drive trains while including the vehicle environment models and the driver. The modularity is developed considering two axes:

- virtual to real : The ability of the developed system ranges from all simulation configuration (SIL) to driver + hardware in the loop simulation (H\&HIL)

- multiplatform: The communication protocol between different systems or models allows easy exchange of systems and simulation platforms (energetic and dynamic vehicle model, different driving simulators configuration, Power plant in a HIL configuration, ...). The chosen protocol, namely the CAN (Control Area Network) can also easily be used to address properly further steps like vehicle prototype design

In the following sections we will describe the developed concepts and tools. The last chapter presents two applications made with the tool. A conclusion lists applications that can be developped with the facility.

\section{TOOL DESCRIPTION}

\section{A. Architecture of the tool}

The main program named MODYVES aims at connecting a generic input and a driver to a generic output with a vehicle model. The main architecture of the framework is presented in figure 1.

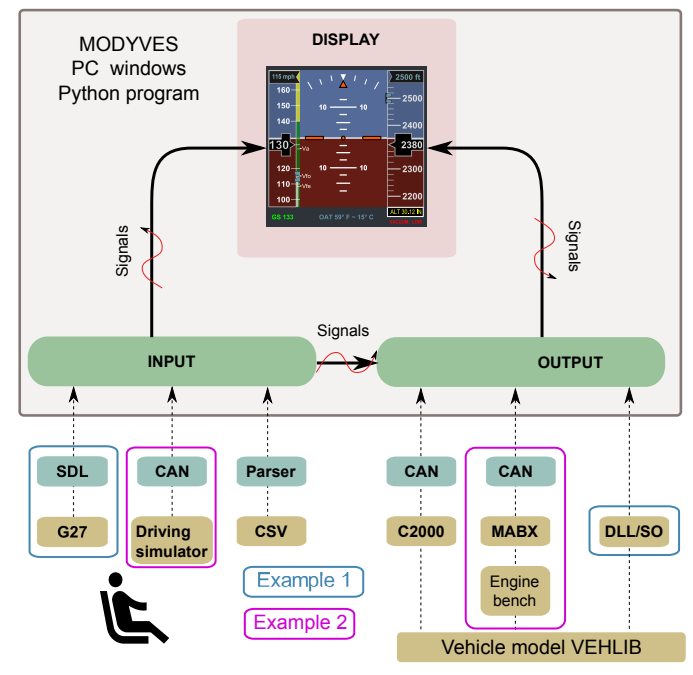

Fig. 1. The Modyves framework 
Inputs can be choosen among:

- a Gamepad like Logitech G27 Joystick or equivalent. Modyves uses Simple DirectMedia Layer ${ }^{1}$ to provide low level access to the device

- IFSTTAR's driving simulator

- a csv file to replay some experiments

Several kind of outputs consisting of the vehicle model are supported:

- Texas Instrument C2000 control card

- $\quad$ dSPACE Hardware with Power Hardware In The Loop like engine test bench

- Dynamic Link Library file

\section{B. Communication layer}

The CAN protocol is widely used in automotive industry. For this reason, we selected this kind of network for the communication of the different pieces of hardware. Easy to implement, another advantage is that the communication can be secured by testing the Rx time (Received frame) in a real time clock.

Two different USB to CAN converter have been integrated in the tool, PEAK ${ }^{2}$ module and Systec ${ }^{3}$ module.

Both provide device driver (Dynamic Link Library files) and header files to connect to the module and parametrise it, decode received frame and send transmitted ones.

\section{Hardware}

Two main hardwares have been integrated in our tool, a prototyping hardware commercialised by dSPACE, namely Micro-autobox (MABX-II), and a low cost 32 bit microcontroler from Texas Instrument, the C2000 Peripheral Experimenter kit equipped with a F28335 control card. Both are supported by The Mathworks with matlab and simulink coder for dSPACE hardware, and matlab and embedded coder for C2000.

The table I makes a short comparison between hardwares.

\begin{tabular}{ccc}
\hline & F28335 & MABX-II $(1401 / 1501)$ \\
\hline Processor & $150 \mathrm{MHz}$ & $900 \mathrm{MHz}$ \\
RAM & $68 \mathrm{kB}(+256 \mathrm{kB} \mathrm{SRAM})$ & $16 \mathrm{MB}$ \\
Flash memory & $512 \mathrm{kB}$ & $16 \mathrm{MB}$ \\
Analog input & $16(12 \mathrm{bits})$ & $16(12 \mathrm{bits})$ \\
Can network & 2 & 4 \\
Analog output & 0 & $8(12 \mathrm{bits})$ \\
PWM & 6 & 4 in, 4 out \\
Bit I/O & 16 DI, 10 DO, 16 shared DIO & 34 shared DIO \\
\hline \multicolumn{2}{c}{ TABLE I. } & HARDWARE CHARACTERISTICS
\end{tabular}

Of course these two devices have not the same performances and are not suitable for the same kind of application.

One can notice the power of dSPACE board (procesor speed and size of RAM and Flash), but the major advantage

\footnotetext{
${ }^{1}$ https://www.libsdl.org/

${ }^{2}$ http://www.peak-system.com/

${ }^{3}$ http://www.systec-electronic.com/
}

of the tool is its combinations with a RTI interface and controlDesk software that enable to rapidly develop, debug and validate a real time project.

On the other hand, TI C2000 control card is well suited for a variety of automotive applications. If offers a good processor speed with a moderate but sufficient Flash memory to developp and embed a real time application at a moderate price. Debugging is far more complex than with dSPACE product.

\section{Software}

Modyves is written in python and is cadenced by a windows timer, so depending upon computer characteristics and computer load, it can deviate from its theorical execution period. Nevertheless, when connected to real plant, the only critical part running in Modyves is the driver behavior and the communication layer.

The IFSTTAR driving simulator is a static driving simulator based on a real Peugeot 308 and a software part named SIM2. The cockpit and all commands are unchanged to offer the most realistic driving environments to the driver. An embedded electronic card in the vehicle reads all sensors values placed on the pedals, the gearbox and the steering wheel. It also controls the force feedback on the steering wheel. The electronic card controls the vehicle dashboard by sending CAN informations through the OBD connector. SIM2 is the IFSTTAR simulator software, and contains various types of models: vehicle, road, sound, traffic and visual models. The road scene is displayed on 5 screens offering up to $180^{\circ}$ horizontal Field Of View. The IFSTTAR driving simulator is used in the field of human factor research, ergonomics studies, energy-efficient driving, advanced training and studies of "Human and Hardware in Loop"( H\&HIL).

VEHLIB is namely a Simulink library. A framework has been developped for years around this library to integrate all the necessary components model to develop and simulate conventional, hybrid or all electric vehicles [7] [8]. VEHLIB is developped with a combined backward or forward approach. The forward models are able to run on real time hardware with their respectives connexion blocks to real environment [9].



Fig. 2. VEHLIB in one H\&HIL configuration

\section{EXAMPLES OF APPLICATION}

The figure 3 presents different step of integration from the pure simulation on a personal computer up to the deployement 
on a final facilty. This procedure has been succesfully conducted for IFSTTAR's driving simulator. For simplicity reason, the vehicle model has been compiled in a C2000 hardware instead of linking the dynamic library to the simulator software.

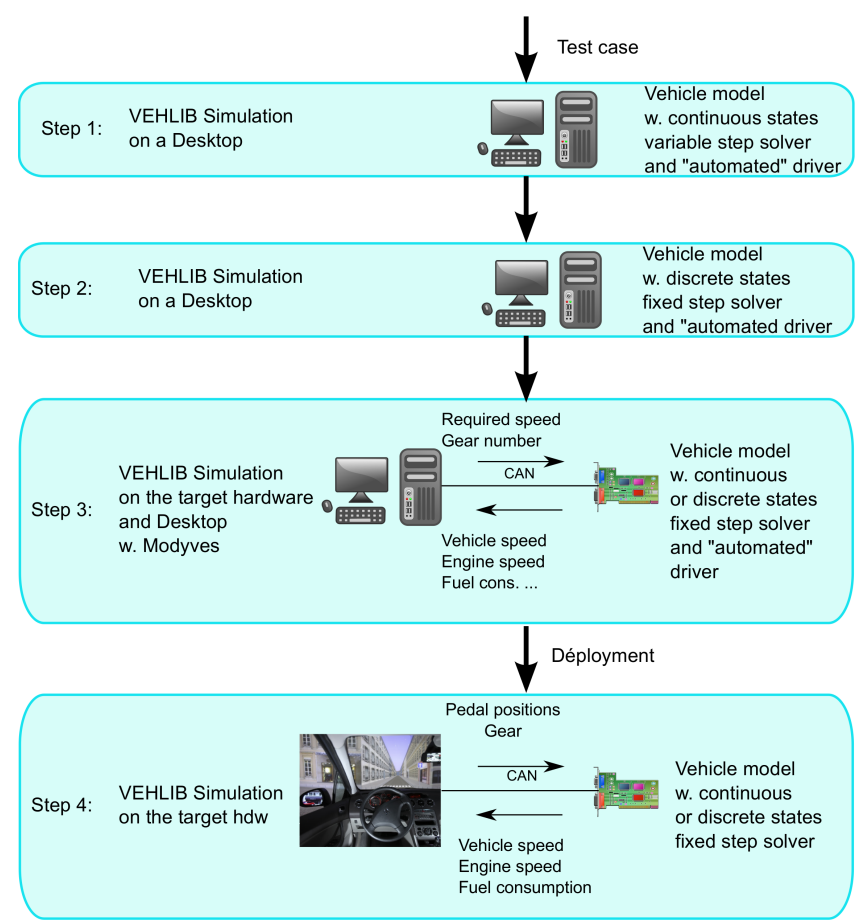

Fig. 3. The different step of integration

As presented in figure 1, the tool allows to make a large variety of tests and integration depending on the effort and the final objective. Two applications are presented hereafter, the first one is a model in the loop application with a G27 joystick, the second is a Power Hardware in the Loop with a driver simulator, a virtual vehicle connected to a real engine. Both include a driver in the loop.

\section{A. G27 Joystick and Model in the loop application}

A first example to introduce the human in the loop consists of a real time application running in a soft real time mode (i.e. a windows timer). It is easy to implement and need only a G27 Joystick and a computer to run (See example 1 in figure II-D).

As mentioned earlier, this application uses a windows timer to set the switching time of the application. In order to verify the real execution period of Modyves, the jitter time has been monitored on a personal laptop (intel core i7 3610QM @ 2.3 GHz running Windows 7) without perturbation (no other program were running on the computer). The theoretical frequency is $1 \mathrm{kHz}$. The results are presented in figure 4 . The mean step time is $0.001002 \mathrm{sec}$., the max value is $0.0027 \mathrm{sec}$. (only one occurence during this test which lasted around 30 seconds) and the minimum value is 0.0009999 second. These values do not affect neither the driver perception, nor the model behavior, because they are far from the time constant of these "systems". Consequently the deviation from the theoretical period is small enough for our application.

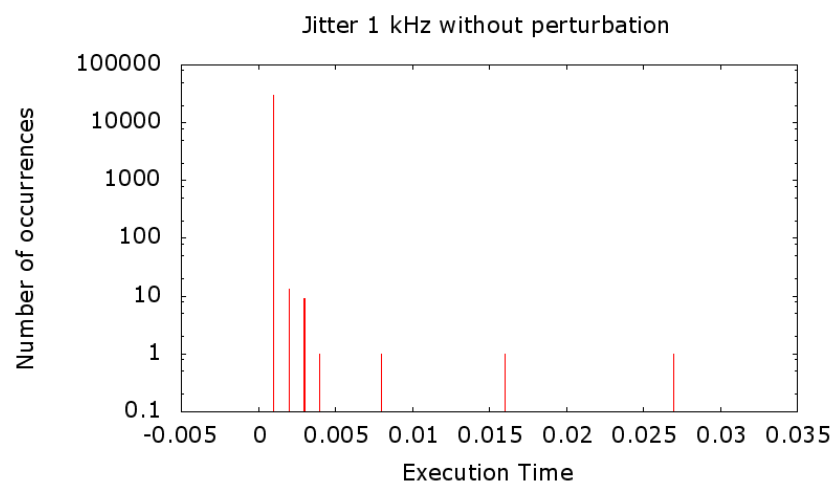

Fig. 4. Jitter for a $1 \mathrm{kHz}$ application

Figures 5 and 6 illustrate the driver behaviour and the model response. The simulated vehicle is a Peugeot $308 \mathrm{SW}$ model equipped with a 5 speed manual gearbox, an EP6

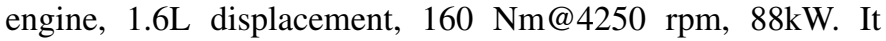
complies EURO IV regulations.
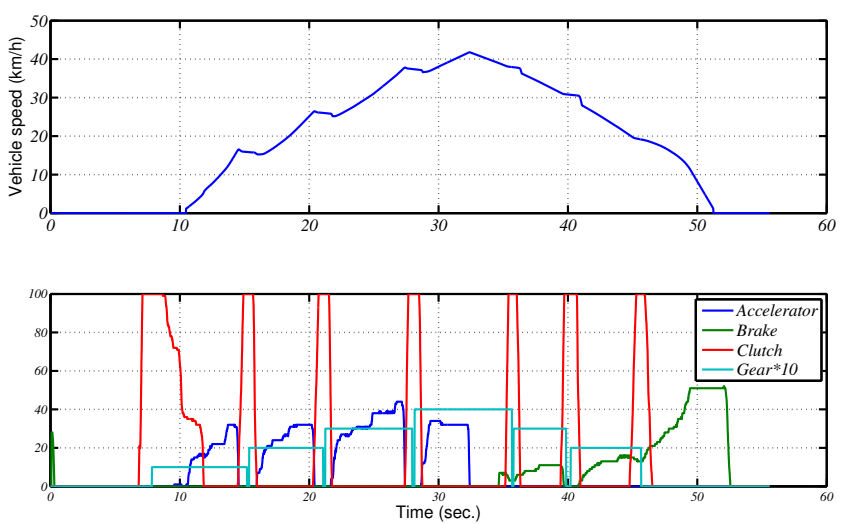

Fig. 5. Vehicle speed and driver actuators
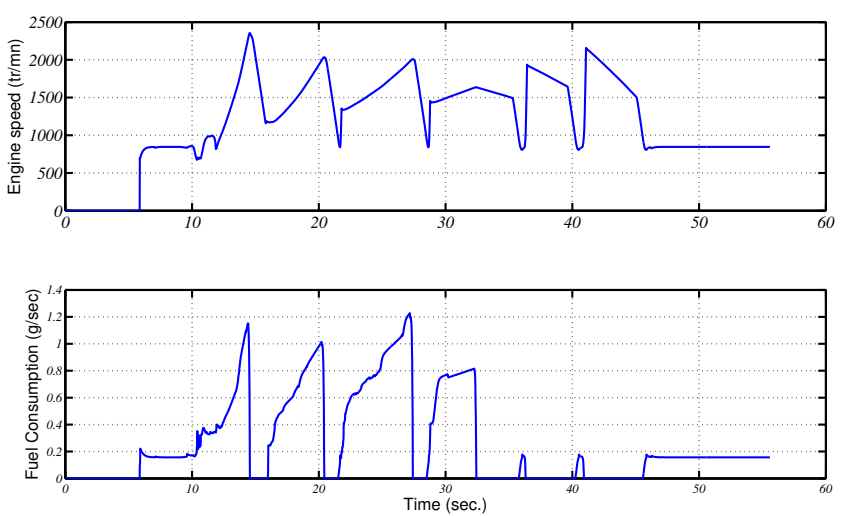

Fig. 6. Simulated engine speed and fuel consumption

\section{B. Driver simulator with HIL application on engine test bench}

This is the most complete situation described in figure II-D. In this case, the vehicle model (excepted the engine) is running in a hard real time platform, a Micro-autobox hardware in this 
particular case. The latter communicates with the engine test bench and exchange some informations with it, namely:

- send accelerator pedal position to the engine and rotation speed to the electric generator

- $\quad$ receive actual torque

The bench is presented in figure 7 .

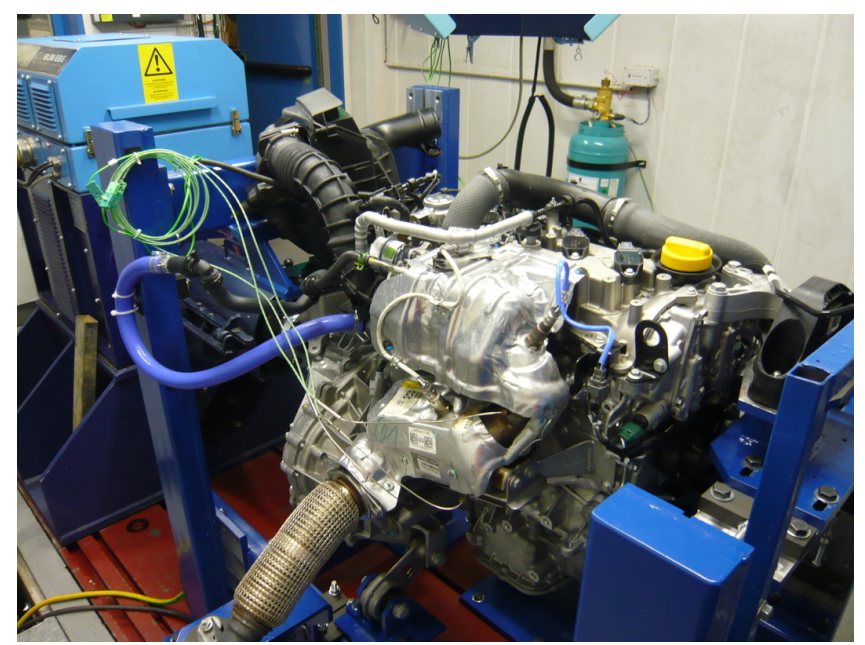

Fig. 7. Engine on the test bench

The bench is running in the so-called throttle/speed mode. At each time step, the actual torque is measured on the bench, transmitted to the model and introduced in the simulated clutch. It passes throwgh the different components up to the vehicle wheels. The longitudinal motion equation is resolved allowing to calculate the speed of the different shafts up to the engine speed, which is in turn transmitted to the bench as a target generator speed. In the same time, the accelerator position is also send to the engine ECU.

The driving simulator is presented in figure 8 .

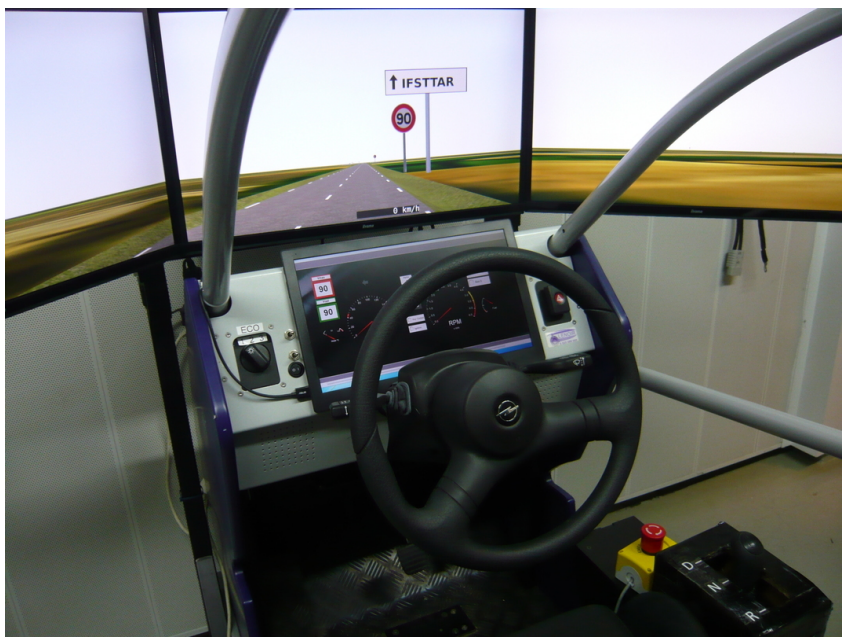

Fig. 8. Driving simulator

Figures 9 and 10 illustrate the behaviour of the driver and the response of the engine on the test bench.
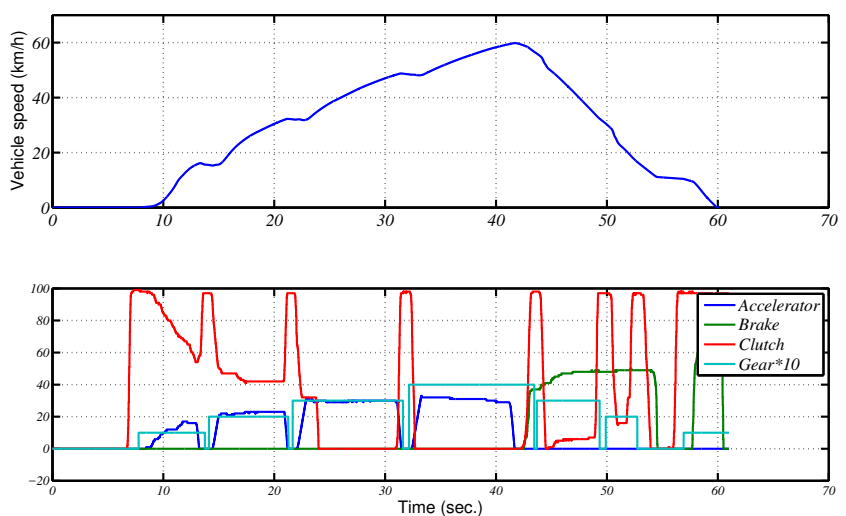

Fig. 9. Vehicle speed and driver actuators
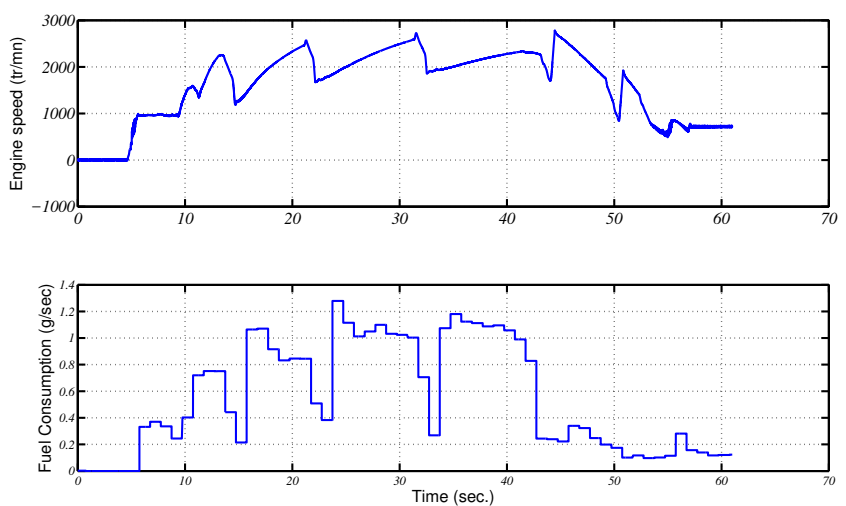

Fig. 10. Measured engine speed and fuel consumption

One can notice that fuel cut-off is effective in engine simulation when the vehicle decelerate (figure 6) but are not present in the bench (figure 10) where cut-off is not validated in the actual engine ECU.

\section{CONCLUSION}

In this paper, a generic framework has been presented to develop and test mecatronic applications in a progressive way. The facility is used in the laboratory to test ADAS (Advanced Driver Assistance Systems) and allows to take into account driver behavior as well as realistic vehicle environment. In this context, not only the fuel consumption is measured in a real engine, but one can measure engine emissions thanks to the gas analyzers available in the laboratory including $\mathrm{CO}, \mathrm{HC}$, NOx and particles measurements.

Number of applications could be performed with this facility:

- the coupled setting could be used to approach a real use behavior of the vehicle and the ICE instead of performing only standard driving cycles. In fact, the new emission regulation consider pollutant measurement on a real track in a procedure called Real Drive Emission (RDE) using Portable devices (PEMS). In order to anticipate this phase, the coupled test bench could help for the engine design and tuning in order to reduce real emissions 
- the facility could embed ADAS system. They can be easily implemented and tested on urban, road or highway context with a human driver in the loop. For example, the assistance systems for eco-driving could be assessed in terms of near-real fuel consumption and pollutant emission

- the simulator can be a platform to evaluate different degrees of driving delegation towards autonomous vehicle. For example, it is easy to simulate manual or automatic gearbox, to test different kind of speed regulator, speed limitation...

- $\quad$ hybrid vehicles can be emulated in the real time model or they can be introduced in the bench to simulate hybrid vehicle. A clutch can also be physically present to test all electric mode for a parallel hybrid vehicle. With this kind of application, several energy management laws can be quickly implemented and tested in a almost real environment

A further step for the software can consist of generalizing it to allow the core module to connect to any kind of devices as described in figure 11 .

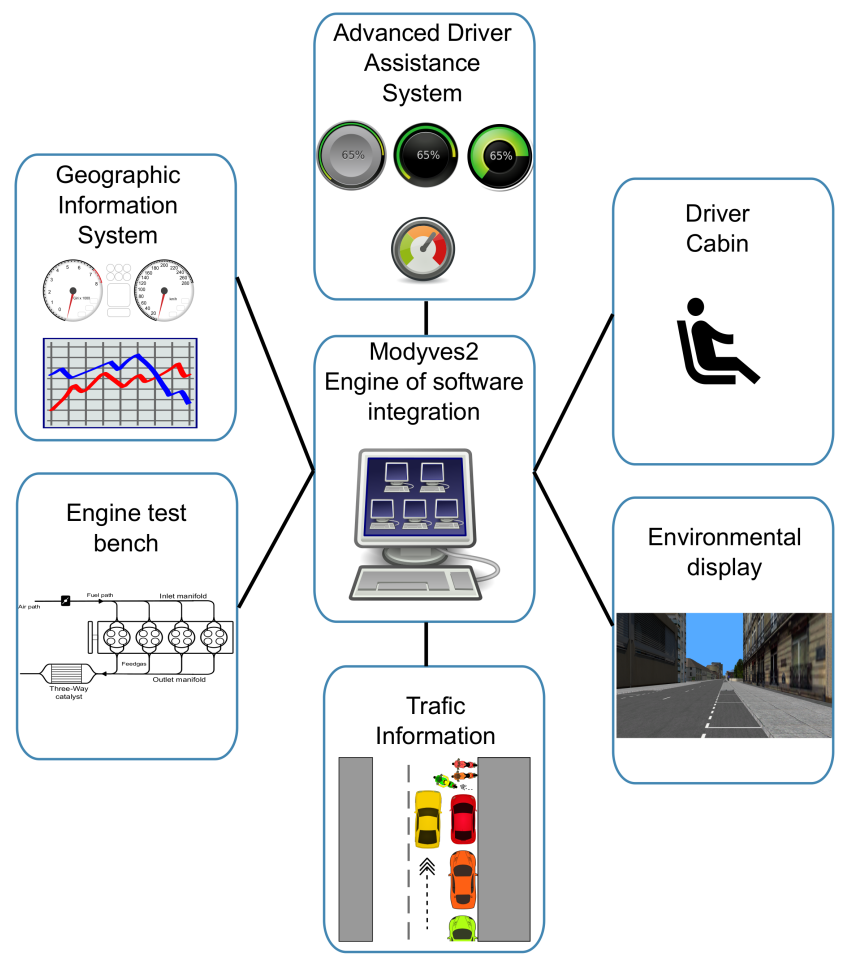

Fig. 11. The engine for software integration

\section{REFERENCES}

[1] S. C. Oh, "Evaluation of motor characteristics for hybrid electric vehicles using the hil concept," IEEE transaction on vehicular technology, 2005.

[2] R. Trigui, B. Jeanneret, B. Malaquin, and C. Plasse, "Performance comparison of three storage systems for mild hevs using phil simulation," IEEE TRANSACTIONS ON VEHICULAR TECHNOLOGY, vol. 58, 2009

[3] A. Bouscayrol, Hardware in the loop simulation in "Control and Mechatronics", J. D. Irwin, Ed. CRC Press 20112011, 2011.
[4] N. Shidore, T. Bohn, M. Duoba, H. Lohse-Busch, and P. Sharer, "Phev 'all electric range' and fuel economy in charge sustaining mode for low soc operation of the jcs vl41m li-ion battery using battery hil," Proceeding of - allcarcentral.com, 2007.

[5] A. Castaings, W. Lhomme, R. Trigui, and A. Bouscayrol, "Comparison of energy management strategies of a battery/supercapacitors system for electric vehicle under real-time constraints," Applied Energy, 2015.

[6] N. Verhille, A. Bouscayrol, P. J. Barre, and J. P. Hautier, "Hardwarein-the-loop simulation of the traction system of an automatic subway," EPE'07, 2007.

[7] E. Vinot, J. Scordia, R. Trigui, and B. Jeanneret, "Model simulation, validation and case study of the 2004 THS of Toyota Prius," Int. J. Vehicle Systems Modelling and Testing, 2008.

[8] B. Jeanneret, R. Trigui, F. Badin, F. Harel, and F. Damemme, "New Hybrid Concept Simulation Tools, evaluation on the Toyota Prius Car," in EVS 16, Oct. 1999.

[9] Jeanneret, Trigui, Malaquin, Desbois-Renaudin, and Badin, "Mise en oeuvre d une commande temps reel de transmission hybride sur banc moteur," Congrés Européen Alternatives énergétiques dans l automobile, 2004. 